\title{
CRISPR-Cas9 as a curative drug for chronic viral infection
}

\author{
Youdiil Ophinni ${ }^{1,2}$ \\ ${ }^{1}$ Ragon Institute of MGH, MIT and Harvard, Cambridge, US \\ ${ }^{2}$ Immunology Frontier Research Center, Osaka University, Osaka, Japan
}

\begin{abstract}
The innovation of CRISPR-Cas9 has single-handedly revolutionized biotechnology by enabling efficient and specific cutting of DNA. CRISPR-Cas9 approaches are promising not only to targetthe human genome but also DNA of pathogenic viruses, which coincidentally is the canonical function in its bacterial origin. Since 2014, a myriad of studies has proven the efficacy of CRISPR-Cas9 treatment to cleave viral DNA intermediates in vitro. One of the most widely targeted is theproviral genome of human immunodeficiency virus type-1 (HIV-1). The disease burden of HIV-1 is massive - the infection is incurable and has remained a pandemic for over four decades. Integrated HIV-1 provirus inside the human genome causes viral persistence inside latent cellular reservoirs, eluding antiretroviral therapy (ART) and sterilizing cure. Specific targeting anddisruption of HIV-1 proviral genome is necessary to achieve viral clearance, which can be achieved with CRISPR-Cas9. Here, we review the features and up to date evidence of CRISPR- Cas9 to target the HIV-1 proviral genome and suppress viral replication. We will also discuss potential CRISPR/Cas9 delivery methods in vivo, combination with other gene editing modalities and other therapeutic approaches, to bring gene editing-based HIV-1 cure closer into clinical use.
\end{abstract}

